\title{
Dalbergia brasiliensis Vogel extracts in vitro antioxidant activity and phytotoxic effect in Lactuca sativa and Allium cepa
}

\author{
Luciane Dalarmi ${ }^{1}$, Cristiane Bezerra da Silva ${ }^{1}$, Ellis Marina \\ Szabo', Daniella Maria Soares de Oliveira', Maislian de Oliveira', \\ Vinícius Bednarczuk de Oliveira', Sandra Maria Warumby Zanin", \\ Josiane de Fátima Gaspari Dias ${ }^{1}$, Obdulio Gomes Miguel ${ }^{1}$, Marilis \\ Dallarmi Miguel ${ }^{1}$
}

${ }^{1}$ Department of Pharmaceutical Sciences, Universidade Federal do Paraná (UFPR), Curitiba, PR, Brazil

\begin{abstract}
Based on medicinal use of Dalbergia species, the present study aimed to evaluate $D$. brasiliensis leaves and barks crude extracts and fractions' antioxidant and phytotoxic potential, employing allelopathic essays and 3 antioxidant essays: Phosphomolybdenum complex reduction, DPPH free radical scavenging and TBARS (lipid peroxidation). Concerning Phosphomolybdenum complex reduction potential, leaves chloroform fraction (LCF) and barks ethyl acetate fraction (BEAF) overcame rutin's reduction potential in $90 \%$ and $23 \%$. DPPH essay revealed leaves ethyl acetate fraction (LEAF) antioxidant potential $\left(\mathrm{IC}_{50}\right.$ de $40,629 \mu \mathrm{g} / \mathrm{mL}$ ), given standards ascorbic acid $\left(\mathrm{IC}_{50}=4,503 \mu \mathrm{g} / \mathrm{mL}\right)$ and rutin $\left(\mathrm{IC}_{50}=7,330 \mu \mathrm{g} / \mathrm{mL}\right)$ activities. TBARS essay demonstrated that leaves hexane fraction (LHF) (51\%) and barks ethyl acetate fraction (BEAF) (41\%) stand out when compared to BHT antioxidant activity $(54 \%)$. Regarding phytotoxicity, the lowest crude extracts and fractions concentration $(250 \mu \mathrm{g} / \mathrm{mL})$ employed were able to inhibit $L$. sativa and A. cepa primary root initial growth, specially leaves hexane fraction (LHF) and leaves chloroform fraction (LCF). Dalbergia brasiliensis fractions might function as stress inhibitors in oxidant systems and alter meristem cellular division, due to present substances' activity.
\end{abstract}

Keywords: Allelopathy, primary root inhibition, antioxidant effect, oxidative stress

\section{INTRODUCTION}

A wide range of plants produce compounds, which, in certain amounts, may provoke toxic effects to surrounding plant species (Harborne, 1994). Allelophaty defines plant interactions employing phytotoxins, once donor plant negatively affects target plant, providing advantage to donor plant (Dayan \& Duke, 2009). Secondary metabolites are able to bring on beneficial and harmful effects, depending on some characteristics such as amount, chemical properties and present functional groups (Barbosa et al., 2005; Goldfarb et al., 2009). Also, are considered as greatly responsible for allelophatic effects observed in nature, since these compounds are important agents against natural predators and ultraviolet radiation, including simple phenolic and heteroside compounds, coumarins, xanthans, tannins, flavonoids, saponins, alkaloids and sulfur compounds (Haida et al., 2010; Jácome et al., 2004).

Dalbergia genus (Fabaceae), comprehends shrubs, creepers and small to medium sized trees, widely distributed in tropical and subtropical regions. Many Dabergia species are employed in Chinese traditional medicine (Carvalho, 2004) and present confirmed biological properties like analgesia, antiinflammatory, antihelminthic, antioxidant, antimicrobial, antiulcerogenic, antitumoral, larvicidal, among others. Genus chemical characteristics are flavonoids, isoflavonoids, proanthocyanidins, xanthonoids, neoflavonoids, glycosides, cinamoil phenols, benzophenones and rotenoids (Vasudeva et al., 2009; Saha et al., 2013). 
Dalbergia brasiliensis, popularly known as jacarandá, caroaba-brava, caviúna or marmeleiro, is distributed among Cerrado regions and Brazilian Atlantic Forest (Carvalho, 2004). Dalbergia brasiliensis research scarcity along Dalbergia genus known biological and pharmacological potential, encouraged antioxidant activity evaluation by three methods (phosphomolybdenum complex, TBARS and DPPH) and allelopathic potential evaluation on Lactuca sativa e Allium cepa inicial growth.

\section{MATERIAL AND METHODS}

\section{Plant material collection}

D. brasiliensis leaves and barks were collected in Curitiba-Paraná (Brazil) 25²6'48.0''S; $49^{\circ} 20^{\prime} 51.2^{\prime \prime} \mathrm{W}$. Plant material taxonomic identification was determined at Municipal Botanic Museum Herbarium (Curitiba) and kept at room temperature for 10 days, until dehydration. Dryed plant material was stored in plastic bags, protected from light and heat.

\section{Crude Ethanolic Extract and Fractions}

Leaves and barks crude ethanolic extracts were obtained in Soxhlet apparatus, employing ethanol $96^{\circ}$ $\mathrm{GL}$, approximately at $78^{\circ} \mathrm{C}$. Crude ethanolic extracts were vacuum filtered (Büchner) and concentrated in rotary evaporator (reduced pressure, $78^{\circ} \mathrm{C}, 150 \mathrm{rpm}$ ). Crude ethanolic extracts were fractioned by liquidliquid partition in modified Soxhlet apparatus (Carvalho et al., 2009) employing solvents in increasing polarity scale, obtaining leaves' and barks' hexane, chloroform and ethyl acetate fractions. Crude ethanolic extracts and fractions were evaporated (water bath, $50^{\circ} \mathrm{C}$ ) and stored in cool dry place.

\section{Antioxidant Activity Evaluation}

Leaves and barks crude ethanolic extracts and fractions had in vitro antioxidant potential evaluation determined employing three different methods.

\section{- Phosphomolybdenum complex reduction}

Samples' ability to reduce phosphomolybdenum complex were evaluated according to Prieto et al. (1999). Samples were diluted in metanol (200 $\mu \mathrm{g} / \mathrm{mL})$. Reagent solution (complex solution) containing sodium phosphate $0,1 \mathrm{M}$ solution $(28 \mathrm{~mL})$, ammonium molybdate $0.03 \mathrm{M}$ solution $(12 \mathrm{~mL})$ and sulfuric acid $3 \mathrm{M}$ solution $(20 \mathrm{~mL})$ had it final volume adjusted to $100 \mathrm{~mL}$. Sample solution was added $(0.3$
$\mathrm{mL})$ to reagent solution $(3 \mathrm{~mL})$ and test tubes were kept sealed in water bath $\left(95^{\circ} \mathrm{C}\right)$ for 90 minutes. After cooling, solutions were analyzed in spectrophotometer (Shimadzu, UV 1601) at $695 \mathrm{~nm}$.

Results showed samples' Relative Antioxidant Activity (RAA\%) when compared to standards (ascorbic acid and rutin) by following equation:

$$
R A A \%=\frac{\text { Abs sample }- \text { Abs blank }}{\text { Abs standard }- \text { Abs blank }} \times 100
$$

- Free Radical Scavenging [2,2-diphenyl-1picrylhydrazyl (DPPH)]

Samples' DPPH scavenging potential were evaluated according to Mensor et al. (2001). Samples and standards (rutin and ascorbic acid) methanolic solutions were obtained (15 to $125 \mu \mathrm{g} / \mathrm{mL}$, except hexane fractions: 100 e $450 \mu \mathrm{g} / \mathrm{mL})$. To each solution $(2.5 \mathrm{~mL})$, DPPH methanolic solution (0.03 $\mathrm{mmol} / \mathrm{mL})$ was added $(1 \mathrm{~mL})$. Blank samples $(2.5$ $\mathrm{mL}$ of sample solution and $1 \mathrm{~mL}$ of methanol) were also evaluated. After thirty minutes, samples, standards and blank samples were analyzed in spectrophotometer $(518 \mathrm{~nm}$, which corresponds to maximum DDPH absorption). Samples' ability to scavenge DPPH was determined as following:

$\% D P P H$ inhibittion $=100-\frac{(\text { Abs sample }- \text { Abs blank }) \times 100}{\text { Abs blank }}$

Samples $\mathrm{IC}_{50}(50 \%$ of inhibitory concentration) were determined by linear regression, establishing an $\mathrm{y}=\mathrm{ax}+\mathrm{b}$ equation, for comparing purposes.

\section{- Thiobarbituric Acid Reactive Substances (TBARS)}

Samples' ability to inhibit lipid peroxidation were evaluated according to adapted Moraes et al. (2006). Samples were diluted in methanol (1000 ppm) and butylated hydroxytoluene (BHT) was employed as standard. Filtered egg yolk $(5 \%)$ in sodium dodecyl sulfate solution $(0.55 \%)$ was employed as lipid source.

Each sample $(1000 \mu \mathrm{L})$ was added to distilled water $(400 \mu \mathrm{L})$ and lipid solution $(500 \mu \mathrm{L})$. Afterwards, to each tube: chlorine 2.2-azo-bis-acid (ABAP) 0.035\% solution $(50 \mu \mathrm{L}), 1.5 \mathrm{~mL}$ of acetic acid $(20 \%, \mathrm{pH} 3.5)$ and thiobarbituric acid (TBA) solution $(0.4 \%)$ were added. Tubes were kept sealed in water bath $\left(95^{\circ} \mathrm{C}\right)$ for an hour. After cooling, n-butanol $(1.5 \mathrm{~mL})$ was added and well mixed, aiming to extract lipid material. Supernatants were collected after centrifugation (3000 rpm, 5 minutes) and analyzed in 
spectrophotometer $(532 \mathrm{~nm})$. Antioxidant Index (AI\%) was determined by following equation:

$$
A I \%=1-\left(\frac{\text { Abs sample }}{\text { Abs standard }}\right) \times 100
$$

\section{Allelopathic activity evaluation}

\section{Germination and growth essays}

D. brasiliensis samples' allelopathic effects were evaluated on lettuce (Lactuca sativa) and onion (Allium cepa) germination and growth. Extracts and fractions solutions (DMSO 1\%) were prepared in 250 $\mu \mathrm{g} / \mathrm{mL}, 500 \mu \mathrm{g} / \mathrm{mL}$ and $1000 \mu \mathrm{g} / \mathrm{mL}$ concentrations. To previously autoclave sterilized $\left(120^{\circ} \mathrm{C}, 20\right.$ minutes) Petri dishes $(9.0 \mathrm{~cm}$ diameter $)$ containing Whatman filter paper $\left(\mathrm{n}^{\mathrm{o}} 1, .0\right)$, samples solutions $(5$ $\mathrm{mL}$ ) were added. Samples and control (sample absence) solutions were buffered by MES (2morpholine ethanesulfonic acid) (10 mM), adjusting $\mathrm{pH}$ (6.0) by adding $\mathrm{KOH} 0.1 \mathrm{~N}$ (Macias et al., 2000).

Each Petri dish was randomly sown (30 seedlings) with Lactuca sativa and Allium cepa, separately in quadruplicates, as described in Brasil (2009). Sown Petri dishes were placed in BOD germination chamber (relative humidity $\pm 80 \%$ and constant temperature: $25^{\circ} \mathrm{C}$ for lettuce and $15^{\circ} \mathrm{C}$ for onion). Lettuce seedlings were incubated in the dark and onion seedlings in 12 hour photoperiod, as suggested in Brasil (2009).

Germination bioessays followed Macias et al. (2000) methodology. Data collection was performed daily for seven days, evaluating radicle protusion (minimum length $2.0 \mathrm{~mm}$ ). Essay conclusion was attested when germination became nule for three consecutive days. Speed Germination Index (SGI) was determined according to Maguire (1962), as shown on following equation:

$S G I=\frac{N \text { germinated seedlings }}{N \text { days of germination }}+\frac{N \text { germinated seedlings }}{N \text { days of germination }}+\cdots$

Growth bioessays followed Barnes et al. (1987) and Macias et al. (2000) described methods. After seven days of radicle protusion, root and hypocotyl/coleoptile were measured over millimiter paper (10 seedlings from each Petri dish).

\section{RESULTS AND DISCUSSION}

Essays performed on D. brasiliensis extracts and fractions, demonstrated fractions' antioxidant activity varied between in vitro methods (Table 1).
Concerning reduction potential, evaluated through phosphomolybdenum complex essay, LCF, BCF and BEAF overcame rutin's activity in $90 \%, 5 \%$ and $23.8 \%$, respectively. LEAF nealy achieved rutin's activity, reaching 93\% of rutin's antioxidant activity. Free radical scavenging (DPPH) essay revealed higher antioxidant potential for LCF $\left(\mathrm{IC}_{50}=42.343\right.$ $\mu \mathrm{g} \mathrm{mL})$ and LEAF $\left(\mathrm{IC}_{50}=40.629 \mu \mathrm{g} \mathrm{mL}\right)$. Extracts and fractions are complex compound blends, therefore rarely will show similar activity as standards ascorbic acid $\left(\mathrm{IC}_{50}=4.503 \mu \mathrm{g} \mathrm{mL}\right)$ and rutin $\left(\mathrm{IC}_{50}=7.330 \mu \mathrm{g} \quad \mathrm{mL}\right)$. TBARS essay demonstrated LHF activity (51\%) as similar to standard BHT (54\%). Data revealed species' antioxidant potential through different mechanisms, confirming Dalbergia medicinal use.

Free radicals or reactive oxygen species are generated by exogenous chemical products or endogenous metabolic processes and are able to oxidize biomolecules, such as nucleic acids, proteins, lipid and DNA. These molecules may initiate different degenerative illnesses like neurological disturbs, cancer, emphysema, cirrhosis, arthritis, atherosclerosis, among others (Halliwell, 2007; Sies, 1997).

Mammals natural antioxidant mechanisms are not always efficient, therefore antioxidant compounds intake becomes an alternative supply, pointing lower rates of antioxidant-related diseases (Antolovich et al., 2002). Supplement ou tea intake protects organism against free radical effects (Szent-Giörgyi, 1963). Many medicinal plants showed antioxidant activity throught pharmacological traditional methods for the past two decades (Kasote et al., 2013). Knowing that, the present study aimed evaluating $D$. brasiliensis antioxidant activity, since other genus species are traditionally known for its antioxidant properties.

Etnophamacologycal and nutraceutical in vitro investigation often employ antioxidant evaluation methods to track and verify plants and its phytochemicals antioxidant potentials. Still, many times, plant extracts oxidant mechanism are difficult to comprehend. Therefore, the present study evaluated antioxidant activity employing methods based on antioxidant mechanism, like DNA damage prevention, lipid peroxidation inhibition or ammonium molibdate reduction (Antolovich et al., 2002).

Table 1. D. brasiliensis effects on antioxidant activity essays 

cepa

\begin{tabular}{l|c|c|c|c}
\hline & \multicolumn{2}{|c|}{ Phosphomolybdenum } & DPPH & TBARS \\
\hline Sample & $\begin{array}{c}\text { Rutin RAA } \\
(\%)\end{array}$ & $\begin{array}{c}\text { Ascorbic Acid } \\
\text { RAA }(\%)\end{array}$ & $\mathrm{IC}_{50}(\mu \mathrm{g} / \mathrm{mL}) \mathrm{x} \pm \mathrm{dp}$ & $\mathrm{AI}(\%)$ \\
\hline LCEE & 45,78 & 11,67 & $72,476 \pm 1,0293$ & 30,16 \\
LHF & 102,81 & 26,22 & $165,602 \pm 2,1437$ & 51,57 \\
LCF & 190 & 48,46 & $42,343 \pm 1,1240$ & 43,21 \\
LEAF & 93,75 & 23,91 & $40,629 \pm 0,1384$ & 36,88 \\
BCEE & 50,93 & 13,23 & $48,303 \pm 0,6064$ & 34,12 \\
BHF & 73,98 & 17,6 & $273,868 \pm 2,1608$ & 33,95 \\
BCF & 105,01 & 24,57 & $43,572 \pm 1,0178$ & 34,7 \\
BEAF & 123,82 & 28,98 & $47,310 \pm 0,1298$ & 41,87 \\
Ascorbic Acid & - & - & $4,503 \pm 0,0507$ & - \\
Rutin & - & - & $7,330 \pm 0,1168$ & - \\
BHT & - & - & - & $54,03 \%$ \\
\hline N & - & - & & \\
\hline
\end{tabular}

Note: LCEE: leaves crude ethanolic extract; LHF: leaves hexane fraction; LFC: leaves chloroform fraction; LEAF: leaves ethyl acetate fraction; BCEE: barks crude ethanolic extract; BHF: barks hexane fraction; $\mathrm{BCF}$ : barks chloroform fraction; BEAF: barks ethyl acetate fraction;

Antioxidant activity essays are performed in medicinal plants in order to evaluate free radical elimination potential, which leads to understanding plants' role in oxidative stress minimizing, as well as related illnesses physiopathology. In this aspect, it is important to state $D$. brasiliensis phytotoxic activity provided by its antioxidant characteristics and interfers in other plants' growth.

Results on lettuce and onion initial growth show $D$. brasiliensis allelopathic potential on seedlings, as growth parameters evaluated on laboratory were altered. All samples in every concentration were able to inhibit lettuce germination period. LHF and BHF provided accentuated delay, when compared to other samples. BAEF provided similar effect to BHF, as shown on Table 2.

Table 2. D. brasiliensis effects on $L$. sativa laboratory germination speed index

\begin{tabular}{|l|c|c|}
\hline \multicolumn{3}{|c|}{ Lactuca sativa Germination Speed Index (GSI) } \\
\hline \multicolumn{3}{|c|}{ Crude ethanolic extracts } \\
\hline $\mathbf{n}=\mathbf{4}$ repetitions -+ & Leaves & Barks \\
\hline Control & $83.33 \pm 2.19$ & $98.00 \pm 0.66$ \\
\hline $1000 \mu \mathrm{g} / \mathrm{mL}$ & $69.48 \pm 3.18^{*}$ & $47.58 \pm 1.17^{*}$ \\
\hline $500 \mu \mathrm{g} / \mathrm{mL}$ & $74.24 \pm 2.23^{*}$ & $49.16 \pm 1.26^{*}$ \\
\hline $250 \mu \mathrm{g} / \mathrm{mL}$ & $87.3 \pm 3.45^{*}$ & $48.81 \pm 2.65^{*}$ \\
\hline \multicolumn{3}{|c|}{ Hexane Fractions } \\
\hline $\mathbf{n}=\mathbf{4}$ repetitions & Leaves & Barks \\
\hline
\end{tabular}




\begin{tabular}{|l|l|l|}
\hline Control & $83.33 \pm 2.19$ & $98.00 \pm 0.66$ \\
\hline $1000 \mu \mathrm{g} / \mathrm{mL}$ & $44.63 \pm 2.47^{*}$ & $47.45 \pm 1.17^{*}$ \\
\hline $500 \mu \mathrm{g} / \mathrm{mL}$ & $47.54 \pm 1.28^{*}$ & $47.80 \pm 2.37^{*}$ \\
\hline $250 \mu \mathrm{g} / \mathrm{mL}$ & $49.54 \pm 1.12^{*}$ & $48.33 \pm 1.22^{*}$ \\
\hline \multicolumn{3}{|c|}{ Chloroform Fractions } \\
\hline $\mathbf{n}=\mathbf{4}$ repetitions & Leaves & Barks \\
\hline Control & $83,33 \pm 2.19$ & $98.00 \pm 0.66$ \\
\hline $1000 \mu \mathrm{g} / \mathrm{mL}$ & $61.66 \pm 1.33^{*}$ & $48.88 \pm 1.17^{*}$ \\
\hline $500 \mu \mathrm{g} / \mathrm{mL}$ & $57.77 \pm 2.37^{*}$ & $47.70 \pm 1.26^{*}$ \\
\hline $250 \mu \mathrm{g} / \mathrm{mL}$ & $64.72 \pm 1.89^{*}$ & $47.62 \pm 2.65^{*}$ \\
\hline \multicolumn{3}{|c|}{ Ethyl acetate Fractions } \\
\hline $\mathbf{n}=\mathbf{4} \mathbf{~ r e p e t i t i o n s}$ & Leaves & Barks \\
\hline Control & $83,33 \pm 2.19$ & $98.00 \pm 0.66$ \\
\hline $1000 \mu \mathrm{g} / \mathrm{mL}$ & $72.36 \pm 2.47^{*}$ & $48.56 \pm 1.17^{*}$ \\
\hline $500 \mu \mathrm{g} / \mathrm{mL}$ & $83.95 \pm 1.28^{\text {ns }}$ & $48.26 \pm 2.37^{*}$ \\
\hline $250 \mu \mathrm{g} / \mathrm{mL}$ & $84.44 \pm 1.12^{\text {ns }}$ & $47.22 \pm 1.22^{*}$ \\
\hline
\end{tabular}

${ }^{1}$ Mean \pm standart deviation. *Treatment mean differs significantly $(\mathrm{P}<0.05)$ when compared to control mean on Dunnet test. ${ }^{\text {NS }}$ Treatment mean does not differ significantly when compared to control mean.

Hormesis effect was observed in onion seedling treated with LCEE and LHF, revealing inhibitory activity in $250 \mu \mathrm{g} . \mathrm{ml}^{-1}$ concentration (75.91 e 74.43, respectively). LCF and LEAF boosted onion seedlings germination speed in all concentrations in a non dose-dependent mean. All BCEE and BEAF concentrations inhibited onion seedlings germination speed and LHF and LCF (both in 500 and 1000 $\mu \mathrm{g} . \mathrm{ml}^{-1}$ ) provided most damages, revealing selective mechanism to monocotyledons (Table 3).

Table 3. D. brasiliensis effects on A. cepa laboratory Germination Speed Index

\begin{tabular}{|l|c|c|}
\hline \multicolumn{3}{|c|}{ Allium cepa Germination Speed Index (GSI) } \\
\hline \multicolumn{3}{|c|}{ Crude Ethanolic Extracts } \\
\hline $\mathbf{n}=\mathbf{4}$ repetitions -+ & Leaves & Barks \\
\hline Control & $41.73 \pm 1.84$ & $64.67 \pm 1.15$ \\
\hline $1000 \mu \mathrm{g} / \mathrm{mL}$ & $45.80 \pm 1.23^{*}$ & $40.45 \pm 1.12^{*}$ \\
\hline $500 \mu \mathrm{g} / \mathrm{mL}$ & $66.82 \pm 1.56^{*}$ & $46.33 \pm 1.00^{*}$ \\
\hline $250 \mu \mathrm{g} / \mathrm{mL}$ & $75.91 \pm 1.07^{*}$ & $34.54 \pm 1.07^{*}$ \\
\hline \multicolumn{3}{|c|}{ Hexane Fractions } \\
\hline $\mathbf{n}=\mathbf{4}$ repetitions & Leaves & Barks \\
\hline Control & $41.73 \pm 1.84$ & $64.67 \pm 1.15$ \\
\hline $1000 \mu \mathrm{g} / \mathrm{mL}$ & $62.19 \pm 1.33^{*}$ & $33.84 \pm 1.05^{*}$ \\
\hline $500 \mu \mathrm{g} / \mathrm{mL}$ & $58.39 \pm 1.17^{*}$ & $33.77 \pm 1.12^{*}$ \\
\hline $250 \mu \mathrm{g} / \mathrm{mL}$ & $74.43 \pm 1.13^{*}$ & $47.29 \pm 1.00^{*}$ \\
\hline \multicolumn{3}{|c|}{ Chloroform Fractions } \\
\hline $\mathbf{n}=\mathbf{4}$ repetitions & Leaves & Barks \\
\hline Control & $41.73 \pm 1.84$ & $64.67 \pm 1.15$ \\
\hline $1000 \mu \mathrm{g} / \mathrm{mL}$ & $75.07 \pm 1.33^{*}$ & $37.60 \pm 1.00^{*}$ \\
\hline $500 \mu \mathrm{g} / \mathrm{mL}$ & $68.63 \pm 2.37^{*}$ & $35.58 \pm 1.12^{*}$ \\
\hline $250 \mu \mathrm{g} / \mathrm{mL}$ & $76.23 \pm 1.89^{*}$ & $43.20 \pm 1.05^{*}$ \\
\hline
\end{tabular}




\begin{tabular}{|l|l|l|}
\hline \multicolumn{3}{|c|}{ Ethyl Acetate Fractions } \\
\hline $\mathbf{n}=\mathbf{4}$ repetitions & Leaves & Barks \\
\hline Control & $41.87 \pm 1.13$ & $64.67 \pm 1.15$ \\
\hline $1000 \mu \mathrm{g} / \mathrm{mL}$ & $74.26 \pm 1.17^{*}$ & $48.03 \pm 1.05^{*}$ \\
\hline $500 \mu \mathrm{g} / \mathrm{mL}$ & $72.86 \pm 1.33^{*}$ & $42.43 \pm 1.13^{*}$ \\
\hline $250 \mu \mathrm{g} / \mathrm{mL}$ & $84.44 \pm 1.32^{*}$ & $41.48 \pm 1.15^{*}$ \\
\hline
\end{tabular}

${ }^{1}$ Mean \pm standart deviation. *Treatment mean differs significantly $(\mathrm{P}<0.05)$ when compared to control mean on Dunnet test. ${ }^{\text {NS }}$ Treatment mean does not differ significantly when compared to control mean.

Concerning primary root, samples' $250 \mu \mathrm{g} / \mathrm{ml}$ concentrations were able to affect lettuce root growth, highlighting LCF (24\%) and BCEE (17\%). Inhibitory effect was also observed in LHF (500 $\mu \mathrm{g} / \mathrm{ml}: 11.1 \%$ and $1000 \mu \mathrm{g} / \mathrm{ml}: 11.44 \%$ ) while LEAF $(1000 \mu \mathrm{g} / \mathrm{ml})$ boosted root growth in $16.15 \%$ (Figure $1 \mathrm{~A}$ and B). BEAF $1000 \mu \mathrm{g} / \mathrm{ml}$ concentration also showed primary root growth inhibition in $L$. sativa in $11 \%$ (Figure 1B).
Hormesis effect was also observed in onion, revealing inhibitory activity in crude ethanolic extracts $250 \mu \mathrm{g} / \mathrm{ml}$ concentrations. Fractions showed similar activity, especially LHF (46\%) and LHF $(42 \%)$. Although inhibitory effect was also observed in barks crude ethanolic extracts, was considered minor when compared to leaves extracts and fractions (Figure 1C and D).

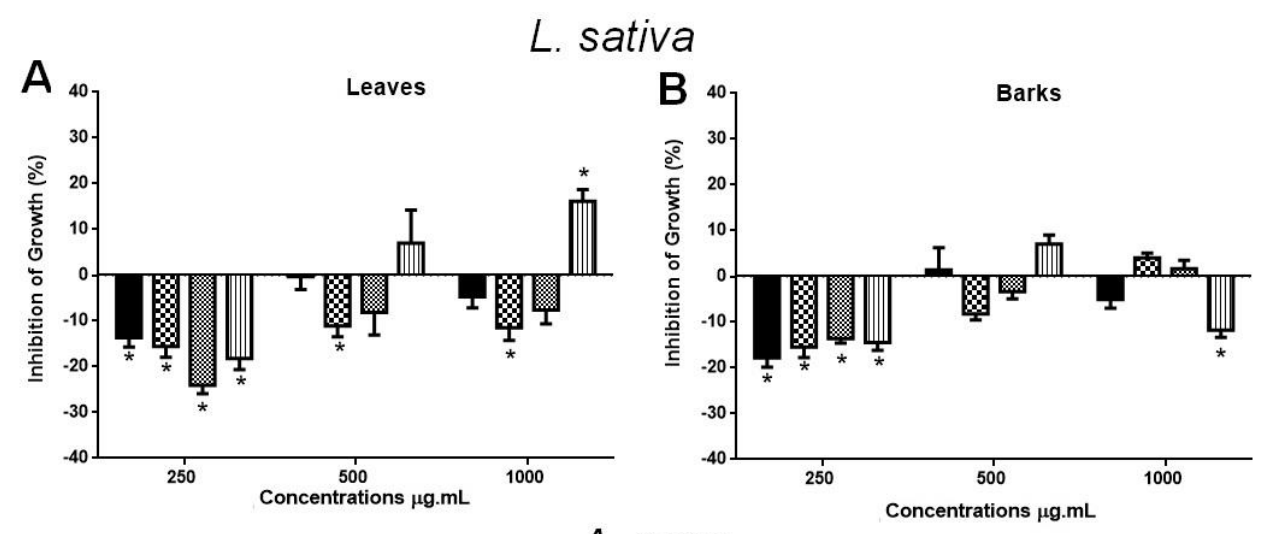

A. cepa
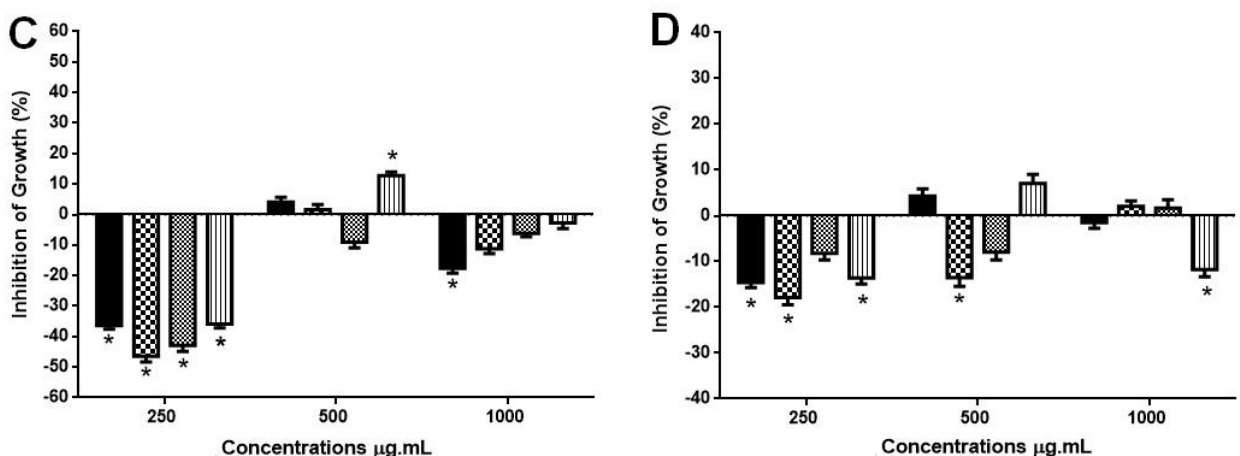

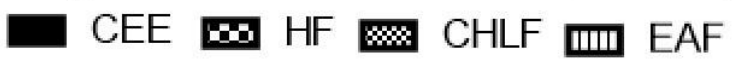

Figure 1. Effects of Crude ethanolic extract (CEE), Hexane fraction (HF), Chloroform fraction (CF) and Ethyl acetate fraction (EAF) of D. brasiliensis, on growth the primary root in leaves (A), barks (B) of $L$. sativa, and growth the primary root in leaves (C), barks (D) of A. cepa. Data expressed in percentage compared to the control. *Statistically different from the control treatment (Dunnett Test, $\mathrm{p}<0.05$ ). 

cepa

Concerning hypocotyl growth in L. sativa, inhibitory effect was also observed in LHF (16.8\%), LEAF $(11.5 \%), \operatorname{BHF}(12.7 \%)$ and $\operatorname{BEAF}(12.8 \%)$ at 250 $\mu \mathrm{g} / \mathrm{ml}$ concentration, revealing phytotoxic activity is not related to extracts concentrations (Figure 2A and B). In A. cepa, $250 \mu \mathrm{g} / \mathrm{ml}$ concentration provided coleoptile inhibition: LCEE (38.4\%), LHF (42\%) and
LEAF (35.8\%), LEAF $500 \mu \mathrm{g} / \mathrm{ml}$ concentration inhibit growth in $46 \%$. BCEE and BCF at $500 \mu \mathrm{g} / \mathrm{ml}$ concentration provoked coleoptiles elongation in $19 \%$ and $58 \%$. All fractions in $250 \mu \mathrm{g} / \mathrm{ml}$ concentration inhibit coleoptiles growth, especially $\mathrm{HF}(35 \%)$ and $\mathrm{CF}(36 \%)$ when compared to control.
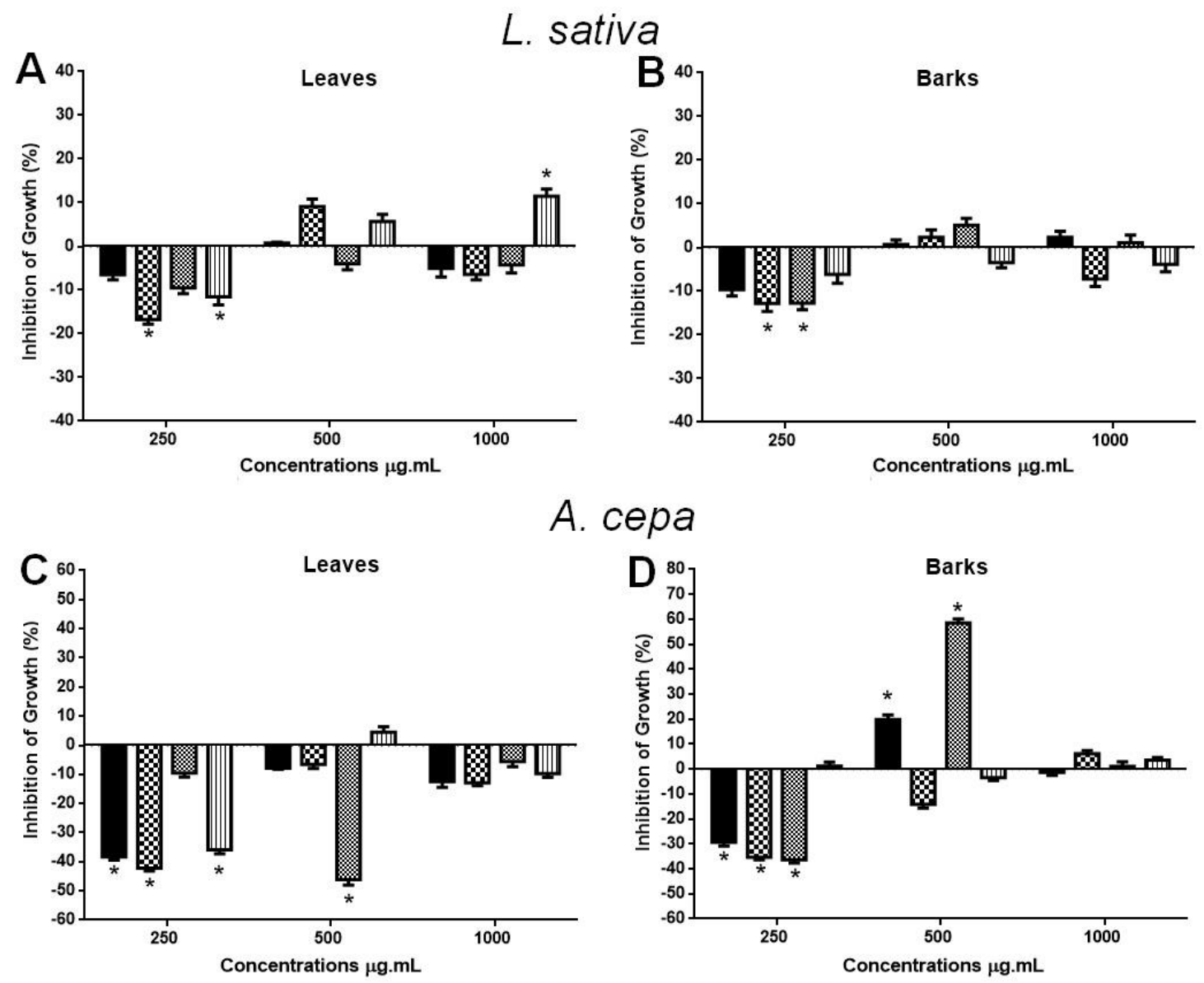

A. cepa

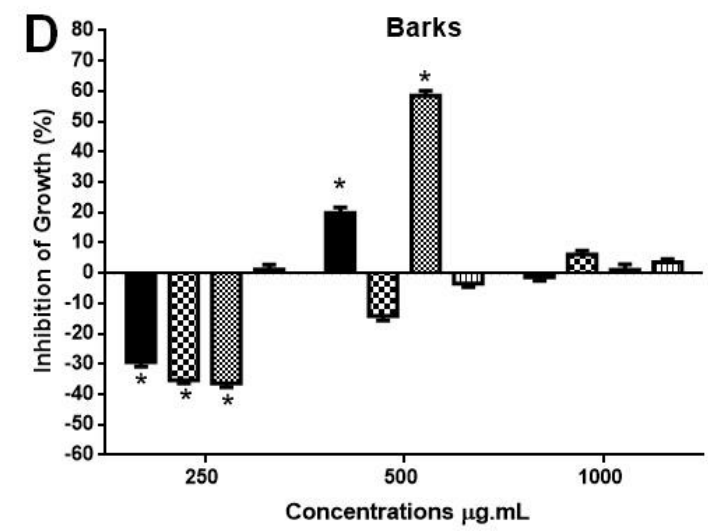

$\square$ CEE $\boldsymbol{\omega} \mathrm{HF}$ \% $\mathrm{m}$ CHLF $\mathbf{m}$ EAF

Figure 2. Effects of Crude ethanolic extract (CEE), Hexane fraction (HF), Chloroform fraction (CF) and Ethyl acetate fraction (EAF) of D. brasiliensis, on growth the Hipocotyl in leaves (A), barks (B) of L. sativa, and growth the coleoptyl in leaves (C), barks (D) of A. cepa. Data expressed in percentage compared to the control. *Statistically different from the control treatment (Dunnett Test, $\mathrm{p}<0.05$ ).

Lipophilic fractions (hexane and chloroform fractions) results might be related to its revealed antioxidant profile. Allelophatic studies have demonstrated that lipidic substances like terpenes may affect cell proliferation and DNA syntesis on plant meristems (Nishida et al., 2005), once mitosis get altered, leading to rampant increase on dividing cells, damaging tubulins, resulting in polyploids nucleus (Hallak et al., 1999; Sanchez-Moreiras et al., 2008).
L. sativa and A. cepa root growth were most affected than hypocotyls and coleoptiles. Baziramakenga et al., (1995) emphasizes that, besides root contact with extracts, root tissues are more permeable to allelochemicals which may alter tissues' initial growth (Nishida et al., 2005), damaging root methabolic activities and cellular division on root extremities that might lead to dry weight reduction (Singh et al., 2003). 
Over the present investigation, negative impact observed on discussed parameters is possibly due to allelochemicals large amounts, which might, significantly or not, have raised stress levels on $L$. sativa e A. cepa. Then, in line with plant activity studies strategies' on evaluating antioxidant and phytotoxic potential, it is advisable to combine in vivo antioxidant assays and toxicity evaluation.

\section{CONFLICTS OF INTEREST}

The authors declared no conflict of interest.

\section{REFERENCES}

I. Antolovich, M.; Prenzler, P.D.; Patsalides, E.; McDonald, S.; Robards, K. Methods for testing antioxidant activity. Analyst., p. 183-98, v.127, 2002.

II. Barbosa, L. C. A.; Maltha, C. R. A.; Demuner, A. J.; Ganem, F. R. Síntese de novas fitotoxinas derivadas do 8-oxabiciclo[3.2.1]oct-6-en-3-ona. Química Nova, p. 444-450, v. 28, n. 3, 2005

III. Barnes; J.P.; Putnam, A.R.; Burke, B.A.; Aasen, A.J Isolation and characterization of allelochemicals in rye herbage. Phytochemistry, p.1385-1390, v.26, n.5, 1987.

IV. Baziramakenga, R.; Leroux, G.D.; Simard R.R. Effects of benzoic and cinnamic acids on membrane permeability of soybean roots. J. Chem. Ecol., p. 12711285, v. 21, 1995

V. Brasil. Ministério da Agricultura e Reforma Agrária. Regras para a Análise de Sementes, SNDA/DNDU/CLU, $2^{\circ}$ Ed. Brasília, 2009.

VI. Carvalho, P.E.R. Jacarandá - Dalbergia brasiliensis. Curitiba: EMPRAPA-CNPF, 2004. (EMBRAPACNPF.Circular Técnica,98)

VII. Carvalho, J.L.S; Cunico, M.M.; Dias, J.F.G.; Miguel, M.D.; Miguel, O.G. Term-stability of extractive processes from Nasturtium officinale R. Br., brassicaceae for Soxhlet modified system. Quim Nova, p. 1031-5, v.32, 2009.

VIII. Dayan, F.E.; Duke, S.O. Biological activity of allelochemicals. Plant-Derived Natural ProductsSynthesis, Function and Application. Dordrecht, The Netherlands: Springer, p.361-384, 2009.

IX. Goldfarb, M; Pimentel, LW; Pimentel, NW. Alelopatia: relações nos agroecossistemas. Tecnol \& Ciên Agropec. [Internet] 2009 [citado 2010 jul. 20];3(1)23-8. Disponível em:

http://www.emepa.org.br/revista/volumes/tca_v3_n1_ fev/tca05_alelopatia.pdf.

X. Haida, K.S.; Coelho, S.R.M.; Costa, J.H.; Viecelli, C.A.; Alekcevetch, J. C.; Barth, E.F. Efeito alelopático de Achillea millefolium L. sobre sementes de Lactuca sativa L. Revista em Agronegócios e Meio Ambiente, p. 101-109, v. 3, n. 1, 2010.

XI. Hallak, A. M. G.; Davide, L. C.; Souza, I. F. Effects of sorghum (Sorghum bicolor L.) root exudates on the cell cycle of the bean plant (Phaseolus vulgaris L.) root. Genet. Mol. Biol., p. 95-99, v.22, 1999.

XII. Halliwell, B. Biochemistry of oxidative stress. Biochem Soc Trans., p. 1147-50, v.35, 2007.
XIII. Harbone, B. The Flavonoids- Advances in Research. since. 1986. Chapman. \&. Hall, London,. U.K.. 1994.

XIV. Jácome, R.L.R.P.; Lopes, D.E.S.; Recio, R.A.; Macedo, J.F.; Oliveira, A.B. Caracterização farmacognóstica de Polygonum hydropiperoides Michaux e P spectabile Mart. (Polygonaceae). Revista Brasileira de Farmacognosia, v. 14, n.1, p. 21-27, 2004.

XV. Kasote, D.M.; Hegde, M.V.; Katyare, S.S Mitochondrial dysfunction in psychiatric and neurological diseases: cause(s), consequence(s), and implications of antioxidant therapy. Biofactors, p. 39206, v.39, 2013.

XVI. Macias, F. A.; Castellano, D.; Molinillo, J. M. G. Search for a standart phytotoxic biossay for allelochemicals. Selection of standard target species. Journal Agricultural and Food Chemistry., p. 25122521, v. 48, n. 6, 2000.

XVII. Maguire, J.D. Speed of germination - aid in selection and evaluation for seedling emergence and vigor. Rev Bras Sementes. [Internet] 1962 [citado 1962 mar.]; v.2(2), p.176-7. Disponível em: http://www.scielo.br/scielo.php?pid=S01013122201000 0300015\&script=sci_arttext.

XVIII. Mensor, L.L.; Menezes, F.S.; Leitao, G.G.; Reis, A.S; Santos, T.C.; Coube, C.S.; Leitao, S.G. Screening of Brazilian plant extracts for antioxidant activity by the use of DPPH free radical method. Phytother Res., p. 127-30, v.15, 2001

XIX. Morais, S.M.; Catunda-Jr, E.A.; Silva, A.R.A; MartinsNeto, J.S. Atividade antioxidante de óleos essenciais de espécies de Croton do nordeste do Brasil. Quim Nova, p. 907-910, v.29, 2006.

XX. Nishida, N.; Tamotsu, S.; Nagata, N.; Saito, C.; Sakai, A. Allelopathic effects of volatile monoterpenoids produced by Salvia leucophylla: inhibition of cell proliferation and DNA synthesis in the root apical meristem of Brassica campestris seedlings.J. Chem. Ecol., p. 1187-1203, v. 31, 2005.

XXI. Prieto, P.; Pineda, M.; Aguilar, M. Spectrophotometric quantitation of antioxidant capacity through the formation of a phosphomolybdenum complex: specific application to the determination of vitamin E. Anal Biochem., p. 337-41, v. 269, 1999.

XXII. Saha, S; Shilpi, J.A.; Mondal, H.; Hossain, F.; Anisuzzman, M.; Hassan, M.M.; Cordell, G.A Ethnomedicinal, phytochemical and pharmacological profile of the genus Dalbergia L. (Fabaceae). Phytopharmacology, v.4, p.291-346, 2013.

XXIII. Sanchez-Moreiras, A.M.; De La Pena, T.C.; Reigosa, M.J. The natural compound benzoxazolin-2(3H)-one selectively retards cell cycle in lettuce root meristems. Phytochemistry, p. 2172-2179, v.69, 2008.

XXIV. Sies, H. Oxidative stress: oxidants, antioxidants. Exp Physiol., p. 291-95, 1997.

XXV. Singh, H.P.; Batish, D.R.; Kohli, R.K. Allelopathic interactions and allelochemicals: new possibilities for sustainable weed management. Crit. Rev. Plant Sci., p. 239-311, v.22, 2003.

XXVI. Szent-Giörgyi, A. Lost in the twentieth century. Annu Rev Biochem., p. 1-15, v.36, 1963.

XXVII. Vasudeva, N.; Vats, M.; Sharma, S.K.; Sardana, S. Chemistry and biological activities of the genus Dalbergia. Pharmacognosy Review, p.307-319, v.3, n.6, 2009. 